\title{
Clinical observation of the effect of modified Ginseng-Schisandra decoction (MGSD) on trace elements and immune function in children with spleen deficiency syndrome after recurrent respiratory tract infection (RRTI): a randomized controlled trial
}

\author{
Hua $\mathrm{Li}^{1}{ }^{\wedge}$, Qing-Ke Cui ${ }^{1}$, Zhan $\mathrm{Li}^{1}, \mathrm{Jie} \mathrm{Li}^{1}$, Feifei $\mathrm{Li}^{2}$ \\ ${ }^{1}$ Department of Chinese Medicine, Shanghai Children's Hospital, Shanghai Jiao Tong University, Shanghai, China; ${ }^{2}$ Shanghai University of \\ Traditional Chinese Medicine, Shanghai, China \\ Contributions: (I) Conception and design: H Li, QK Cui; (II) Administrative support: H Li, J Li; (III) Provision of study materials or patients: H Li, Z \\ Li, J Li; (IV) Collection and assembly of data: H Li, QK Cui, F Li; (V) Data analysis and interpretation: QK Cui, F Li; (VI) Manuscript writing: All \\ authors; (VII) Final approval of manuscript: All authors. \\ Correspondence to: Qing-Ke Cui. Doctor of Medicine, attending physician. Department of Chinese Medicine, Shanghai Children's Hospital, Shanghai \\ Jiao Tong University, Shanghai, China. Email: fishlook123@sohu.com.
}

Background: Recurrent respiratory tract infection (RRTI) is common disease in childhood, and the incidence rate is increasing in China. The cause is complex, which is related to the physiological characteristics of children's respiratory system and the immature immune function, lack of trace elements and environmental pollution. Immunomodulators and antibiotics are often used to treat RRTI, but the clinical efficacy still needs to be improved. Chinese medicine has unique advantages in the treatment of RRTI. In the clinical treatment of RRTI, the authors often use modified Ginseng-Schisandra decoction (MGSD). In order to study the clinical effect of MGSD on serum trace elements and immune function in children with spleen deficiency syndrome after RRTI, we conducted this clinical observation.

Methods: This is a single-center randomized trial with two parallel groups. Eighty children who developed spleen deficiency syndrome following RRTI were randomly and equally divided into a control group and an observation group. The control group was given Medilac-vita and the observation group was given MGSD. The participants, care givers, and those assessing the outcomes were blinded to group assignment. The treatment course was 4 weeks. Clinical efficacy, traditional Chinese medicine (TCM) syndrome scores, humoral immunity, and trace element index values before and after treatment were compared between the two groups of children.

Results: The trial was finished. The total effective rate of the observation group ( $\mathrm{n}=40,95 \%$ ) was significantly higher than control group $(n=40,65 \%)(\mathrm{P}<0.05)$. After treatment, the TCM syndrome scores of the two groups were reduced compared to those before treatment, while the serum immunoglobulin A and $\mathrm{G}$ levels were increased $(\mathrm{P}<0.05)$; however, the observation group had significantly lower TCM syndrome scores than the control group. Significant improvements in serum immunoglobulins (IgA and IgG) levels, T lymphocyte subsets $\left(\mathrm{CD}^{+}, \mathrm{CD}^{+}\right.$, and $\left.\mathrm{CD}^{+} / \mathrm{CD}^{+}\right)$, and trace elements $(\mathrm{Zn}$ and $\mathrm{Fe})$ indicators were observed in the observation group compared to the control group $(\mathrm{P}<0.05)$.

Conclusions: The application of MGSD in the treatment of children with spleen deficiency syndrome after RRTI has a definite clinical effect. MGSD can effectively improve the immune function of the patients and the content of the trace elements iron and zinc, and thus has potential value for promotion and application.

Trial registration: Chinese Clinical Trial Registry ChiCTR2100047128.

Keywords: Recurrent respiratory tract infection (RRTI); spleen deficiency syndrome; modified GinsengSchisandra decoction (MGSD); clinical efficacy

^ ORCID: Hua Li, 0000-0002-1193-0452; Qing-Ke Cui, 0000-0002-0483-8383. 
Submitted May 18, 2021. Accepted for publication Jun 17, 2021.

doi: $10.21037 /$ tp-21-243

View this article at: https://dx.doi.org/10.21037/tp-21-243

\section{Introduction}

Recurrent respiratory tract infection (RRTI) refers to the frequent occurrence of upper or lower respiratory tract infections during a 1-year period, which exceeds a certain range (1). In China, pediatric respiratory infections account for about $60 \%$ of outpatient cases, of which $30 \%$ are recurrent respiratory infections, and the incidence is rising (2). Modern medical treatment for RRTI mostly involves the use of immunomodulators for immune enhancement of patients with primary immunodeficiency, and antibiotics and other anti-infective therapies for those with infection indications (1). However, for some children, the curative effect is poor, and improper dosage of immunomodulators can increase adverse reactions and suppress immune function (3).

Failure to prevent and treat RRTIs in time can have longterm effects, which can cause damage to the transportation function of the spleen and stomach. "Spleen deficiency syndrome after infection in children" is a traditional Chinese medicine (TCM) syndrome which entails symptoms similar to "spleen deficiency syndrome", including loss of appetite, fatigue and sweating, irregular stools, weight loss, and a pale complexion (4). These symptoms and signs are often delayed and repeated, and can even last for up to several years, causing adverse effects on the growth and development of children.

According to TCM theory, children's bodies are made up of "tender Yin and tender Yang", and the lung and spleen, in which RRTIs are located, are often insufficient. The internal and external causes of RRTI are "deficiency type" and "exogenous evil", respectively. The two factors interact with each other, resulting in insufficient chemogenesis and easy infection by external pathogens.

In modern medical treatment, antibiotics should be used as anti-infective treatment of bacterial infections. However, in TCM, antibiotics are believed to be cold and cool (5), with their long-term application further weakening the spleen and stomach, making it difficult to repeatedly cure the patient. Therefore, replenishing Qi, invigorating the spleen, strengthening the defense of disease, and nourishing the lung are the key to treatment (6). Clinical practice has shown that for children with chronic illness and chronic cough, the use of TCM for strengthening the spleen and body, and nourishing Qi can effectively improve the immune function of children, maintain the steady state and normality of the intestinal flora, and prevent spleen deficiency syndrome, while outperforming antibiotics or probiotics alone.

In recent years, the authors have used modified GinsengSchisandra decoction (MGSD) to invigorate the spleen and replenish Qi in order to treat children with spleen deficiency syndrome after RRTI, which has achieved a good clinical curative effect. The present study aimed to observe the effect of MGSD on serum trace elements and immune function in children with spleen deficiency syndrome after RRTI. We present the following article in accordance with the CONSORT reporting checklist (available at https:// dx.doi.org/10.21037/tp-21-243).

\section{Methods}

This was a single-center randomized trial with two parallel groups and allocation ratio was 1:1. It was conducted to study the clinical effect of MGSD on serum trace elements and immune function in children with spleen deficiency syndrome after RRTI. A total of 80 children with spleen deficiency syndrome after RRTI admitted to the Department of Traditional Chinese Medicine of Shanghai Children's Hospital between June 2019 and May 2020 were enrolled by the staffs of this study. The children were randomly divided into an observation group and a control group, with 40 cases in each group with simple randomization. The participants, care givers, and those assessing the outcomes were blinded to group assignment. The sample size calculation method is as follows: According to the relevant literature and calculation formula. All procedures performed in this study involving human participants were in accordance with the Declaration of Helsinki (as revised in 2013). The study was approved by the ethics committee of Shanghai Children's Hospital (Children's Hospital Affiliated to Shanghai Jiaotong University) (No.: 2015R017-F02) and informed consent was taken from all the patients.

\section{Case selection}

The inclusion criteria for study participants were as follows: 
(I) diagnosed with RRTI in accordance with the standards formulated by the scientific branch of the Chinese Medical Association (7); (II) diagnosed with spleen deficiency syndrome in accordance with the relevant TCM literature standards (4); and (III) boys or girls aged 1-12 years old; and (IV) informed consent was obtained from the patient's family.

The exclusion criteria were: (I) patients with serious diseases such as heart, brain, liver, kidney, or hematopoietic system diseases; (II) patients with repeated respiratory infections caused by tuberculosis, foreign bodies in the bronchus, congenital diseases; and (III) patients with mental illnesses.

\section{Treatment methods}

Children in the control group were treated with Medilacvita (live combined Bacillus subtilis and Enterococcus faecium granules with multivitamins, Beijing Hanmei Pharmaceutical Co., Ltd., approval no. National Medicine Standard 30002270S). The usage and dosage conditions are as follows: for children under 2 years old: 1 bag each time, 1 or 2 times a day, with warm water; and for children over 2 years old: 1 or 2 bags each time, 1 or 2 times a day, with warm water. The treatment course lasted 4 weeks.

Children of treatment group were taken MGSD. The basic prescription consists of: Radix astragali $(6 \mathrm{~g})$, Codonopsis pilosula (6 g), Atractylodes macrocephala Koidz. (6 g), Poria cocos (9 g), Schisandra (3 g), Ophiopogon japonicus $(6 \mathrm{~g})$, oyster (15 g), Radix Glycyrrhizae Preparata (3 g), and Saposhnikovia divaricata $(10 \mathrm{~g})$. Prescriptions of Chinese medicine should be added or subtracted based on the patient's clinical symptoms. The MGSD for children is given as decoction-free granules (Jiangyin Tianjiang Pharmaceutical Co., Ltd.). The usage is as follows: 1 to 2 years old: 2 times/day, 1/3 doses/time; 3 to 6 years old; 2 times/day, 0.5 doses/time; 6 years old and above, 2 times/day, 1 dose/time. The treatment course lasted 4 weeks. The two drugs are packaged in a lightproof package and are similar in appearance.

\section{Primary endpoints}

\section{TCM syndrome scores}

Before and after treatment, the patients were assessed using a TCM syndrome scoring method. The main symptoms (loss of appetite, nausea and vomiting, excessive sweating and fatigue, and loose stools) were given a score of $0,2,4$, or 6 points (none, mild, moderate, or severe, respectively).

\section{Clinical efficacy}

After the course of treatment, the clinical efficacy was determined with reference to the relevant standard efficacy in the "Guiding Principles for Clinical Research of New Chinese Medicines". Clinical efficacy was evaluated as follows: (I) clinical recovery: TCM syndrome score reduction $\geq 95 \%$; (II) significantly effective: TCM syndrome score reduction $\geq 70 \%$; (III) effective: TCM syndrome score reduction $\geq 30 \%$; and (IV) ineffective: TCM syndrome score reduction $<30 \%$.

\section{Secondary endpoints}

\section{Laboratory indicators}

The children's laboratory indicators were examined before and after treatment including serum immunoglobulin levels, $\mathrm{T}$ lymphocyte subsets, and trace element indicators.

\section{Statistical analysis}

SPSS (Statistical Product and Service Solutions, IBM) 21.0 software was used for all statistical analysis of the data in this study. Measurement data were expressed as means \pm standard deviations $(\bar{x} \pm \mathrm{s})$ and were analyzed using $t$-tests, while rank data were analyzed using the rank-sum test. $\mathrm{P}<0.05$ indicated that a difference was statistically significant.

\section{Results}

The treatment period of this clinical observation was 4 weeks. The control group was given the treatment with Medilac-vita (live combined Bacillus subtilis and Enterococcus faecium granules with multivitamins, Beijing Hanmei Pharmaceutical Co., Ltd., approval no. National Medicine Standard 30002270S). The treatment group was treated with MGSD. After 4 weeks, the clinical efficacy, serum immunoglobulin, T-lymphocyte subsets and trace elements were recorded and observed. After 4 weeks, the treatment was finished after the recovery, no medication was needed, and the treatment plan was changed if the condition was not cured. The trail was already finished. The participant flow diagram was shown (Figure 1).

\section{Demographic of patients}

In the observation group, there were 23 males and 17 females, aged 3-12 years old, with an average of $6.50 \pm 2.81$ years old; 


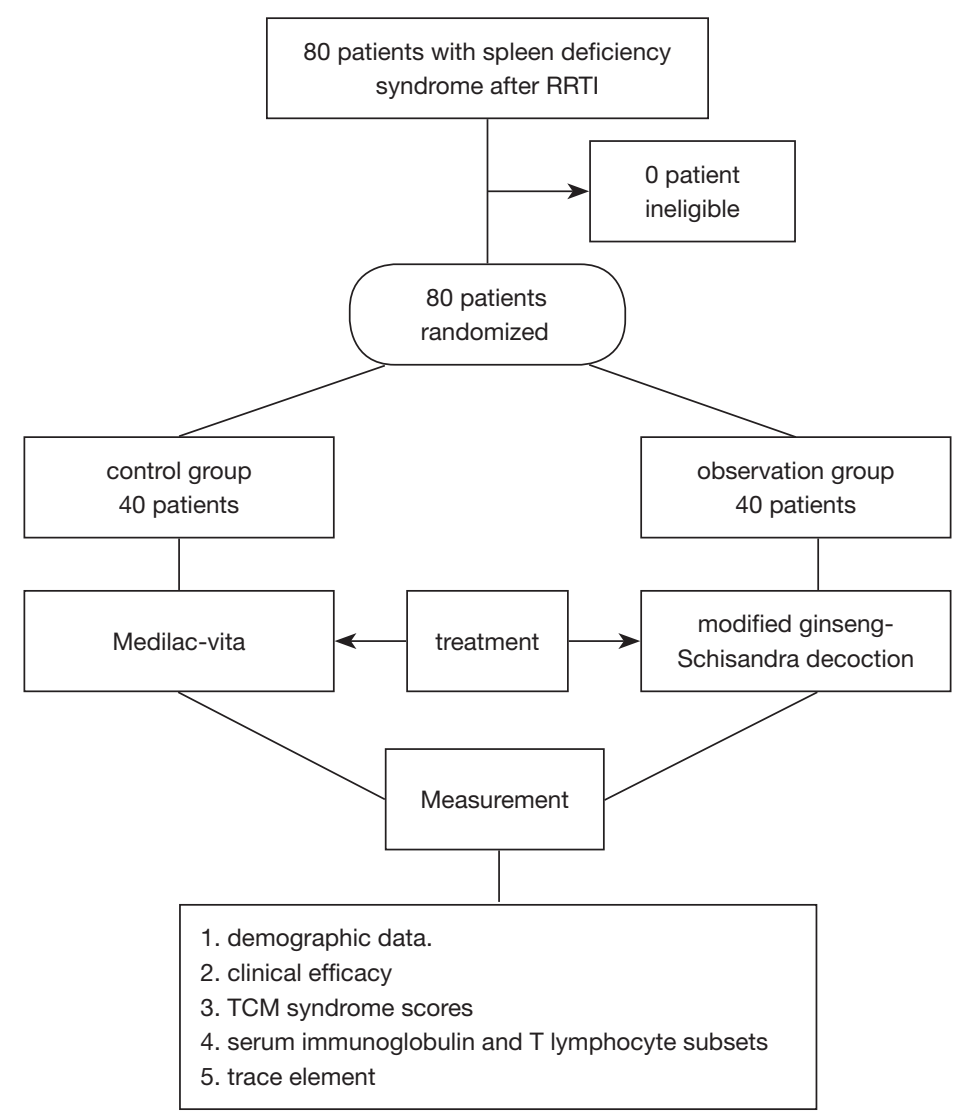

Figure 1 Participant flow diagram. RRTI, recurrent respiratory tract infection.

in the control group, there were 28 males and 12 females, aged 3-12 years old, with an average of $6.83 \pm 2.26$ years old. No statistically significant differences were found in baseline data between the two groups $(\mathrm{P}>0.05)$, meaning they were comparable (Table 1).

\section{Comparison of clinical efficacy}

The total effective rates in the observation and control groups were $95 \%$ and $65 \%$, respectively, which translated to a statistically significant difference in efficacy between the two groups $(\mathrm{P}<0.05)$ (Table 2$)$.

\section{Comparison of TCM syndrome scores}

Before treatment, the TCM syndrome scores in the two groups were not statistically significantly different. After treatment, the TCM syndrome scores in both groups were lower than those before treatment. The TCM syndrome scores of the observation group were significantly lower than those of the control group $(\mathrm{P}<0.05)$ (Table 3).

\section{Changes in humoral immune indexes}

In both groups, the levels of serum immunoglobulin A, $G$, and $M$ were significantly increased after treatment compared with before treatment $(\mathrm{P}<0.05)$; however, the levels of serum immunoglobulin $A$ and $G$ in the observation group were significantly higher than those in the control group $(\mathrm{P}<0.05)($ Table 4).

\section{Changes in the indicator values of trace elements}

Compared with those before treatment, the levels of the serum trace elements $\mathrm{Fe}, \mathrm{Zn}$, and $\mathrm{Ca}$ were increased in the two groups after treatment, while those of $\mathrm{Pb}$ were decreased $(\mathrm{P}<0.05$, Table 5). After treatment, the levels of $\mathrm{Fe}$ and $\mathrm{Zn}$ in the observation group were higher than those in the control group, and the levels of $\mathrm{Pb}$ were lower than those in the control group $(\mathrm{P}<0.05$, Table 5). There was 
Table 1 Demographic of patients

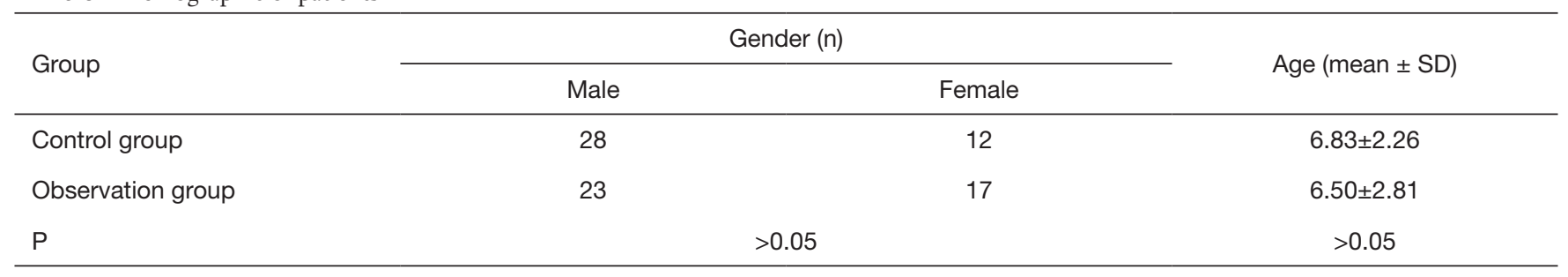

Table 2 Comparison of clinical efficacy/cases

\begin{tabular}{lcccccc}
\hline Group & No. of cases & Clinical recovery & Significantly effective & Effective & Ineffective & Total effective rate (\%) \\
\hline Observation & 40 & 15 & 16 & 7 & 2 & 95.0 \\
Control & 40 & 8 & 7 & 11 & 14 & 65.0 \\
\hline
\end{tabular}

Compared with before treatment, $\mathrm{P}<0.05$.

Table 3 Comparison of TCM syndrome scores before and after treatment

\begin{tabular}{lccc}
\hline Group & No. of cases & Before treatment & After treatment \\
\hline Observation & 40 & $15.85 \pm 2.50^{\mathrm{a}, \mathrm{b}}$ & $3.00 \pm 2.60^{\mathrm{a}, \mathrm{b}}$ \\
Control & 40 & $16.30 \pm 3.22^{\mathrm{a}}$ & $7.25 \pm 2.01^{\mathrm{a}}$ \\
\hline
\end{tabular}

${ }^{\mathrm{a} C}$ Compared with before treatment, $\mathrm{P}<0.05$; ${ }^{\mathrm{b}} \mathrm{Compared}$ with the control group after treatment, $\mathrm{P}<0.05$.

Table 4 Comparison of changes in serum immunoglobulin and $\mathrm{T}$ lymphocyte subsets

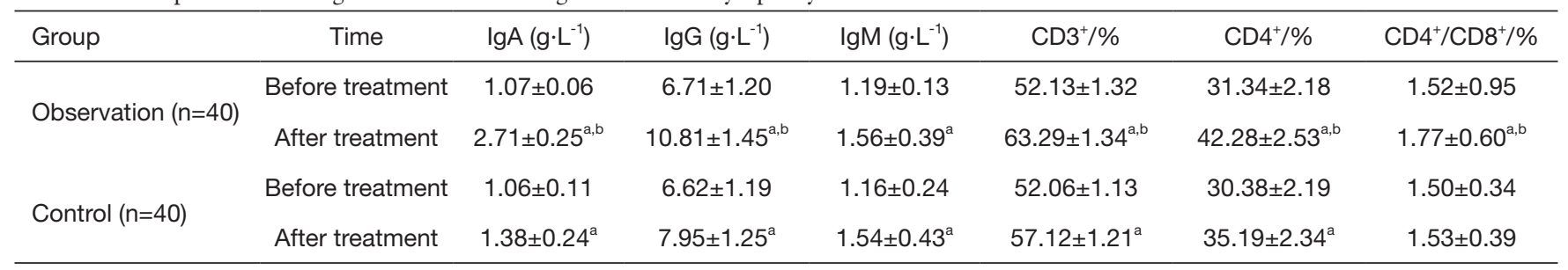

${ }^{\mathrm{a} C}$ Compared with before treatment, $\mathrm{P}<0.05$; ${ }^{\mathrm{b}}$ Compared with after treatment, $\mathrm{P}<0.05$.

Table 5 Comparison of changes in trace element index values $\left(\mu \mathrm{mol} \cdot \mathrm{L}^{-1}\right)$

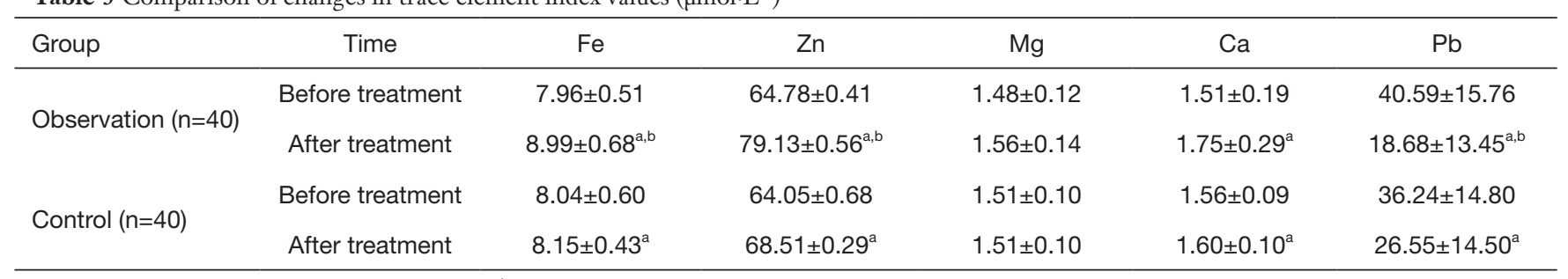

${ }^{\mathrm{a} C}$ Compared with before treatment, $\mathrm{P}<0.05$; ${ }^{\mathrm{b}} \mathrm{C}$ mpared with the control group after treatment, $\mathrm{P}<0.05$. 
no significant difference in the $\mathrm{mg}$ index before and after treatment in either of the groups $(\mathrm{P}>0.05)$.

\section{Discussion}

RRTI is a common and frequently occurring disease in childhood, which is related to multiple factors, including immune function and respiratory physiological characteristics. RRTI damages immune function and increases susceptibility, thus forming a vicious circle $(8,9)$. The term "spleen deficiency syndrome after pediatric infection" was first put forward by Zhongfa Meng, a wellknown TCM pediatrician. It refers to a combination of symptoms similar to "spleen deficiency syndrome" after one or more acute or subacute infections $(4,10)$.

According to TCM, the etiology of spleen deficiency syndrome in children following infection mainly includes weakness of the spleen and stomach, inadequate diet or medication, insufficient rest, and other factors. The spleen and stomach are the foundation of acquired nature, reservoir of food and drink, and the source of Qi and blood biochemistry. If the body is weak in the spleen and stomach or feels external evils, such as eating disorders, cold and heat disorder, or overwork, it can affect the rise and fall of the spleen and stomach Qi, As time goes on, the function of transportation and metabolism will be affected and the spleen and stomach will be weak. Further, if the disease is prolonged or cold and evil, it can cause weakness of the spleen and even the spleen-yang deficiency.

Studies have reported that children with spleen deficiency syndrome often display the following pathophysiological changes: decreased serum gastrin content, reduced the value of bentiromide test (BT-PABA), accelerated gastrointestinal emptying during exercise, gastrointestinal flora disorder, and increased detection rates of pathogenic bacteria and conditional bacteria, as well as relative hyperactivity of parasympathetic nerve function (11). Based on this, we can infer that spleen deficiency syndrome is a debilitating disease state, the main manifestations of which are reduced secretion, excretion, absorption, and motor function of the gastrointestinal tract, neurohumoral and immune regulation disorders, and low nutritional metabolism. The resulting mechanism is gastrointestinal weakness, hypofunction, or disorder.

The intestinal mucosa is the largest immune organ in the human body (12), and the intestinal mucosal immune barrier constitutes the first line of defense in the body's immune system, which can prevent pathogenic microorganisms from entering the body and produce specific immune responses (13). In the state of spleen deficiency, abnormal changes occur in the intestinal mucosal immune barrier. In rats with spleen deficiency, the duodenal mucosa is injured, and the expression of secretory $\operatorname{IgA}(\operatorname{sg} A)$ in the ileum and cecum decreases (14). As the main effector factor of local immune response in the intestinal mucosa, sIgA can prevent the adhesion and colonization of pathogens in the intestine (15); it can not only neutralize and coat toxic substances by combining with complement and lysozyme, but can also prevent bacterial translocation, resist protein hydrolysis, and participate in the defense of the gastrointestinal mucosa. Therefore, the level of sIgA can directly reflect the local immune state of the body's mucosa (16).

Ginseng-Schisandra decoction originates from "Youyou Jicheng", written by Fuzheng Chen during the Qing Dynasty. The original work states: "It is also a magical prescription to treat long-term cough and deficiency of Qi and weak Qi, white face and white lips.” The application of this prescription for the treatment of spleen deficiency syndrome is intended to replenish the foundation of acquired nature. If the spleen and stomach are healthy, water and soil transportation is normal, the visceral organs are provided with sufficient nutrition, the blood circulation is vigorous, and the body's resistance to disease is enhanced; thus, the body can recover from sickness easily. The lung belongs to gold; the spleen belongs to earth; and the earth generates gold. Only when the spleen and earth are strong, can the lung be raised, and cough and asthma be cured; this theory underpins the method of cultivating earth to generate gold.

The MGSD used in the observation group in this study is a combination of Ginseng-Schisandra decoction, Sijunzi decoction, and Shengmaiyin. It's a prescription with which the author has had regular clinical experience. Codonopsis pilosula and Radix astragali are principal drugs belonging to the spleen and lung meridian. Codonopsis pilosula has the effects of tonifying the middle and invigorating Qi, invigorating the spleen and lungs, invigorating the blood and activating fluid, and eliminating dysphoria. It can enhance the immune function, regulate the intestinal tract, reduce the absorption of inflammatory mediators and toxins, and has a good blood-replenishing effect (17). Radix astragali is able to enter the spleen and stomach, and is an important medicine for tonifying the middle and Qi. It can tonify the lung and spleen, replenish Qi, and consolidate the exterior. Atractylodes macrocephala Koidz. combined with Poria cocos can strengthen the spleen, dry 
dampness, and replenish Qi. Schisandra with Ophiopogon japonicus has the functions of nourishing Yin and moistening the lung, benefiting the stomach and promoting body fluid, clearing away the heart fire, and eliminating irritability. Saposhnikovia divaricata has the functions of dispelling wind, relieving the surface, and eliminating dampness. According to modern pharmacological research, Saposhnikovia divaricata has antipyretic and analgesic, antiinflammatory, anti-bacterial, and anti-allergen properties, and can regulate immune function (18). All of the above drugs are minister drugs.

TCM holds the belief that oysters have the functions of submerging yang and replenishing Yin, and reconciling Yin and Yang; their combination means that "Righteousness is stored in the body, and evil cannot be dried." Modern research has found that oysters' chemical composition has calcium carbonate, iron, zinc, and other trace elements, and that it is rich in protein-zinc and has a wide range of pharmacological activities (19), which can enhance the body's defense against non-specific stimuli. Radix Glycyrrhizae Preparata can tonify the spleen, replenish Qi, and harmonize various medicines. The above two drugs are advanced and dispatcher drugs.

The MGSD has the effects of nourishing the Qi of spleen and stomach, nourishing Yin, and moisturizing the lungs. Based on the theory that "all diseases are based on Qi of stomach and spleen deficiency", focusing on prevention of diseases before they occur, and usually focusing on prenatal prevention and diet care (20), based on the promotion of the spleen and digestion, combined with TCM acupoint application. Tuina and other combined internal and external therapies have unique advantages in improving symptoms, eliminating pathogens, and strengthening the foundation, as well as regulating physical fitness; therefore, they can significantly enhance the body's immunity and achieve the purpose of treating diseases $(21,22)$.

T lymphocyte subsets differ significantly between children with RRTI and healthy children (23), and along with serum trace elements, they are closely related to RRTIs $(24,25)$. The results of this study showed that the total effective rate in the observation group was $95 \%$, which was significantly higher than the $65 \%$ observed in the control group $(\mathrm{P}<0.05)$. Furthermore, the improvements in TCM syndrome score, serum immunoglobulin A and G levels, T lymphocyte subgroup levels, and the trace elements $\mathrm{Zn}$ and Fe were greater in the observation group than in the control group (all $\mathrm{P}<0.05$ ).

There are many components of MGSD, and network pharmacological analysis confirmed that the top three TCM monomer molecules are quercetin, kaempferol and isoflavone (our unpublished data). Quercetin has been reported to treat myocardial infarction in a clinic trial (26). Kaempferol is also the active ingredient of ginkgo biloba, detecting the level of kaempferol in urine could reflect the intake of ginkgo biloba (27). Isoflavone has been used in treating menopause-related osteoporosis (28).

Although some achievements have been made in this study, it also has some limitations. For example, the sample size of clinical research needs to be further increased. The clinical observation of multi center and big data was not done. In addition, there are not many indicators for clinical observation, which need to be further increased. Finally, the principle of randomized controlled trials should be further followed.

These data show that MGSD can significantly improve the clinical symptoms and immune function of children with post-RRTI spleen deficiency syndrome, as well as increase the content of $\mathrm{Fe}$ and $\mathrm{Zn}$, with fewer adverse reactions and a higher safety profile than western medicine. Therefore, it has potential in-depth research and application value.

\section{Acknowledgments}

Funding: This project was supported by Shanghai Municipality's Three-year Action Plan for Further Accelerating the Development of Chinese Medicine (2018-2020) (grant No. ZY[2018-2020]-FWTX-4023), and Shanghai Municipal Health Commission's Maternal and Child Health TCM Special Construction Project (grant No. FYJKZY-EB-2).

\section{Footnote}

Reporting Checklist: The authors have completed the CONSORT reporting checklist. Available at https://dx.doi. org/10.21037/tp-21-243

Data Sharing Statement: Available at https://dx.doi. org/10.21037/tp-21-243

Conflicts of Interest: All authors have completed the ICMJE uniform disclosure form (available at https://dx.doi. org/10.21037/tp-21-243). The authors have no conflicts of interest to declare.

Ethical Statement: The authors are accountable for all aspects of the work in ensuring that questions related 
to the accuracy or integrity of any part of the work are appropriately investigated and resolved. All procedures performed in this study involving human participants were in accordance with the Declaration of Helsinki (as revised in 2013). The study was approved by the ethics committee of Shanghai Children's Hospital (Children's Hospital Affiliated to Shanghai Jiaotong University) (No.: 2015R017-F02) and informed consent was taken from all the patients.

Open Access Statement: This is an Open Access article distributed in accordance with the Creative Commons Attribution-NonCommercial-NoDerivs 4.0 International License (CC BY-NC-ND 4.0), which permits the noncommercial replication and distribution of the article with the strict proviso that no changes or edits are made and the original work is properly cited (including links to both the formal publication through the relevant DOI and the license). See: https://creativecommons.org/licenses/by-nc-nd/4.0/.

\section{References}

1. Jiang Z, Shen K, Shen Y, et al. Practice of Pediatrics. 8th. Beijing: People's Medical Publishing House 2015;1288-90.

2. Jiang $\mathrm{Y}$, Zhang Q. Practical Pediatrics of traditional Chinese medicine. Shanghai Sci \& Tech Publisher 1995:455

3. Xu Y, Huang H. Effect of Shenqi Wuweizi Granule on immune function in children with recurrent lower respiratory tract infection. World J Integr Tradit West Med 2019;38:1885-7.

4. Meng Z. Diagnosis and Treatment of Infantile Spleen Asthenia Syndrome after Infection. Shanghai J Tradit Chin Med 2000;34:12-4.

5. Yu Z, Wang L. Observation on the curative effect of Renshen Wuweizi decoction combined with probiotics kucaojijun granules in the treatment of spleen deficiency syndrome after recurrent respiratory tract infection. Nei Mongol J Tradit Chin Med 2019;38:70-2.

6. Su T, Zhao A. The Clinical Effect of Modified Ginseng and Schisandra Decoction in the Treatment of Children With Recurrent Respiratory Tract Infection. CHSM 2019;22:128-30.

7. Group of Respirology, Chinese Pediatric Society, Chinese Medical Association, Editorial board of Chinese Journal of Pediatrics. Clinical Concept and Treatment Principle of Recurrent Respiratory Tract Infection. Chin J Pediatr 2008;46:108-10.

8. Pan L, Li R, Wu S, et al. Clinical effect of Saccharomyces boulardii on treatment of children with recurrent respiratory tract infection .Chin J Nosocomiology 2020;30:2095-9.

9. Lee $\mathrm{E}$, Yoon J, Cho HJ, et al. Respiratory reactance in children aged three to five years with post infectious bronchiolitis obliterans is higher than in those with asthma. Acta Paediatr 2017;106:81-6.

10. Meng Z. Discussion on Differentiation and Treatment of Spleen Deficiency Syndrome in Children after Infectionan Analysis of 404 Cases. J Tradit Chin Med 1988;35-7.

11. Geng W, Li X, Zhao M, et al. Efficacy and Safety Analysis of Hericium Erinaceus Extract Granules in the Treatment for Spleen Deficiency Syndrome after Infection in Children. World Chinese Medicine 2017;12:2036-8.

12. Liu J, Lin X, Wang M, et al. Discussion on the theory of "spleen as the guard" based on intestinal mucosal immunity. J Basic Chin Med 2013;19:460-6.

13. Morris A Ps Tawil A, Bericova Z, et al. Junctional Adhesion Molecules (JAMs) are differentially expressed in fibroblasts and co-localize with ZO-1 to adherens-like jimctions. Cell Commim Adhes 2006;13:233-47.

14. Macpherson A J, Gatto D, Sainsbuiy E, et al. A primitive T cell-independent mechanism of intestinal mucosal IgA responses to commensal bacteria. Science 2000;288:2222-6.

15. Su F, Patel GB, Hu S, et al. Induction of mucosal immunity through systemic immunization: Phantom or reality. Hum Vaccin Immunother 2016;12:1070-9.

16. Wu X, Zhou L, Luo X, et al. Effect of polysaccharide of Sijunzi Decoction on gut flora and immune function in spleen deficiency mice. Pharm Clin Chin Materia Medica 2014;30:12-4.

17. Li X. Survey of research on the chemical constituents and pharmacological effects of Codonopsis. Chin Rural Med Pharm 2014;83-4.

18. Xin G, Li X, Huang X. Research progress on chemical constituents and pharmacological action of radix sileris. Jilin J Tradit Chin Med 2018;38:1323-5.

19. Zhang L, Sha F, Cui G, et al. Progress in pharmacological research of schisandra lignans. J Guiyang Coll Tradit Chin Med 2014;36:55-8.

20. Liang Q. Research progress on prevention and nursing of children with repeated respiratory infections. Huaxia Med 2016;29:189-92.

21. Deng Y, Yang X, Zhang X. Overview of research on treatment of children with repeated respiratory tract infections with external TCM therapy. Guangxi University of Tradi Chin Med 2017;20:55-9. 
22. Chen H, Xie M, Li Y, et al. A Discussion on Treating Children with Recurrent Respiratory Tract Infections Based on "Activating Spleen and Promoting Digestion". Clin Med \& Engin 2016;23:1427-8.

23. Lu Y, Peng G, Lin Q, et al. Comparative analysis of T lymphocyte subsets of children with recurrent respiratory tract infections from 1 to 6 years old and healthy children. Practical Med Tech 2020;27:302-30 .

24. Pan X, Liu H, Liang M, et al. The relationship between serum trace elements and peripheral blood T lymphocyte subsets and children with recurrent respiratory tract infections. J Clin Med Pract 2014;18:51-3 .

25. Tao S. Analysis of the lack of serum trace and macro elements in 200 children with recurrent upper respiratory tract infections and the prevention and treatment effects of intermittent zinc and iron supplementation. Chin Mater \&
Child Health Care 2017;32:1498-501 .

26. Dehghani F, Sezavar Seyedi Jandaghi SH, Janani L, et al. Effects of quercetin supplementation on inflammatory factors and quality of life in post-myocardial infarction patients: A double blind, placebo-controlled, randomized clinical trial. Phytother Res 2021;35:2085-98.

27. Wang FM, Yao TW, Zeng S. Determination of quercetin and kaempferol in human urine after orally administrated tablet of ginkgo biloba extract by HPLC. J Pharm Biomed Anal 2003;33:317-21.

28. Sansai K, Na Takuathung M, Khatsri R, et al. Effects of isoflavone interventions on bone mineral density in postmenopausal women: a systematic review and metaanalysis of randomized controlled trials. Osteoporos Int 2020;31:1853-64.
Cite this article as: $\mathrm{Li} \mathrm{H}$, Cui QK, Li Z, Li J, Li F. Clinical observation of the effect of modified Ginseng-Schisandra decoction (MGSD) on trace elements and immune function in children with spleen deficiency syndrome after recurrent respiratory tract infection (RRTI): a randomized controlled trial. Transl Pediatr 2021;10(6):1692-1700. doi: 10.21037/tp21-243 